\title{
Primary Extramedullary Plasmacytoma of Maxillary Sinus
}

\author{
Chinmaya Sundar Ray, Aparajita Mishra, Rabindra Kumar Khatua, Prasenjit Baliarsingh
}

\begin{abstract}
A rare case of extramedullary plasmacytoma of maxillary sinus in a 58-year-old man presenting with right-sided nasal obstruction and intermittent blood tinged nasal crusting. Nasal endoscopy revealed that a dark red colored mass arising from the lateral wall of nose. A biopsy specimen was diagnosed as plasmacytoma (Kappa light chain type). Serum and urine electrophoresis failed to detect any myeloma component or Bence-J ones protein. All other screening tests to rule out multiple myeloma were negative. These findings confirmed the diagnosis of extramedullary plasmacytoma. The mass was completely excised by lateral rhinotomy approach. No recurrence has been noted at the end of 6 months follow-up.
\end{abstract}

Keywords: Extramedullary plasmacytoma, Kappa light chain, Lateral rhinotomy, Multiple myeloma.

How to cite this article: Ray CS, Mishra A, Khatua RK, Baliarsingh $P$. Primary Extramedullary Plasmacytoma of Maxillary Sinus. Clin R hinol An Int J 2013;6(1):51-53.

\section{Source of support Nil}

Conflict of interest: None declared

\section{INTRODUCTION}

Plasmacytomas are malignant tumors of monoclonal plasma cells. They originate either in bone (solitary osseous plasmacytoma) or in soft tissues (extramedullary plasmacytoma). ${ }^{1}$ Extramedullary plasmacytoma is a rare localized tumor. It has no characteristic of multiple myeloma, but the development of multiple myeloma has been noted in 8 to $36 \%$ of patients. ${ }^{2,3}$

N early $80 \%$ of all extramedullary plasmacytomas occur in head and neck; most of these arise in upper aerodigestive tract, probably because of the abundance of the lymphatic tissues in this area. ${ }^{4,5}$ Extramedullary plasmacytomas represent less than $1 \%$ of all head and neck malignancies ${ }^{6}$ and it accounts for $4 \%$ of all nonepithelial tumors of the nasal cavity, nasopharynx and paranasal sinuses. ${ }^{7}$ W e report a rare case of extramedullary plasmacytoma arising from the maxillary antrum.

\section{CASE REPORT}

A 58-year-old male attended ENT OPD with history of rightsided nasal obstruction and intermittent blood tinged nasal discharge since 6 months and mild proptosis. $\mathrm{N}$ asal endoscopy revealed a dark red colored mass arising from the lateral wall of right side nasal cavity. L eft nasal cavity and nasopharynx were normal. No pal pable cervical lymph nodes. Computed tomographic (CT) scan of PNS revealed a heterogeneous moderately enhancing soft tissue, density lesion involving right side maxillary sinus extending to right side nasal cavity, ethmoid sinus and orbital floor (Figs 1A and $B$ ). Few areas of bony erosion are seen in floor of orbital wall, inferior turbinate, middle turbinate. Biopsy was taken through nasal endoscopy which on histopathological examination reported to be plasmacytoma (Fig. 2).

Further investigations were undertaken to look for multiple myeloma. These investigations included a CBC, serum calcium and creatinine levels, serum protein electrophoresis and immunoelectrophoresis. U rine analysis for Bence-J ones protein, evaluation of $\beta_{2}$ microglobulin, a skeletal survey with plain films (skull, ribs, humerus and femur), abdominal ultrasonography, bone marrow aspiration and biopsy. All findings were within normal limits. Accordingly, we ruled out multiple myeloma and this confirmed diagnosis of primary extramedullary plasmacytomas of maxillary sinus.

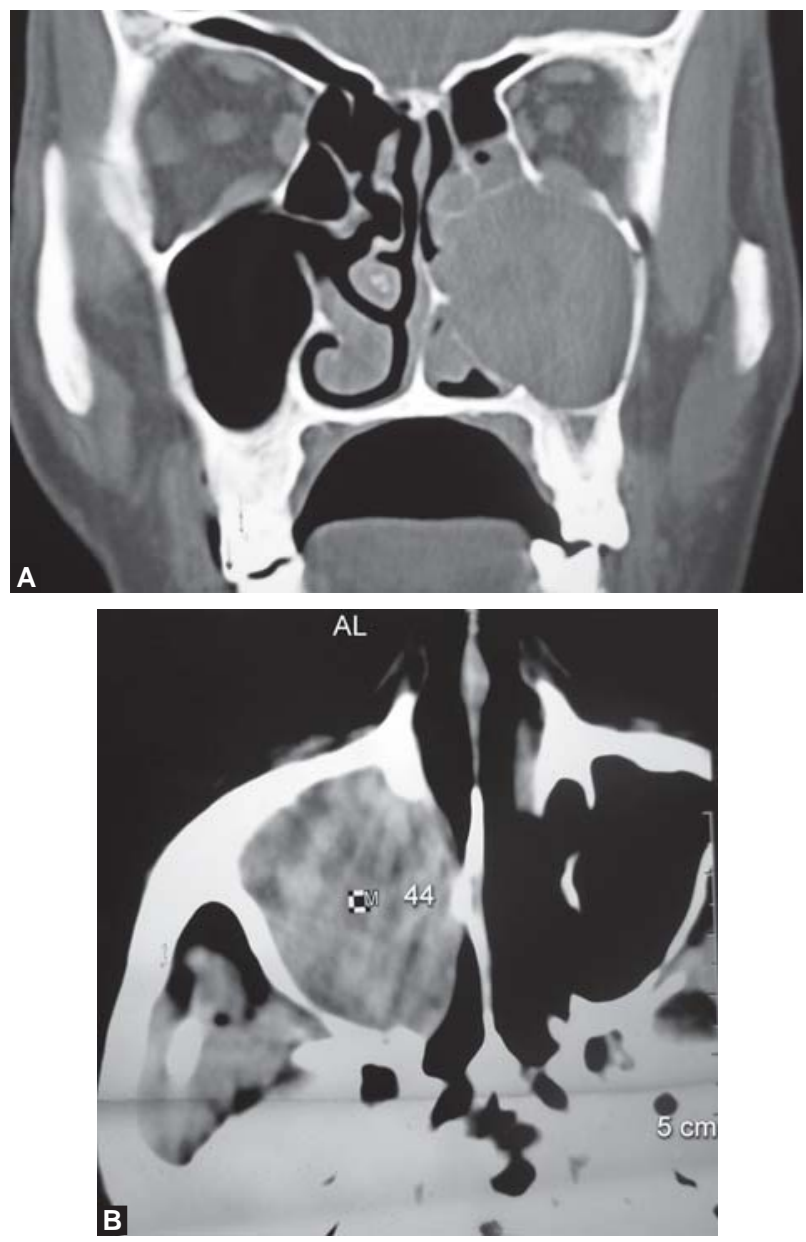

Figs $1 \mathrm{~A}$ and $\mathrm{B}$ : Computed tomographic (CT) scan of PNS showing a heterogeneous moderately enhancing soft tissue, density lesion involving right side maxillary sinus extending to right side nasal cavity, ethmoid sinus and orbital floor 


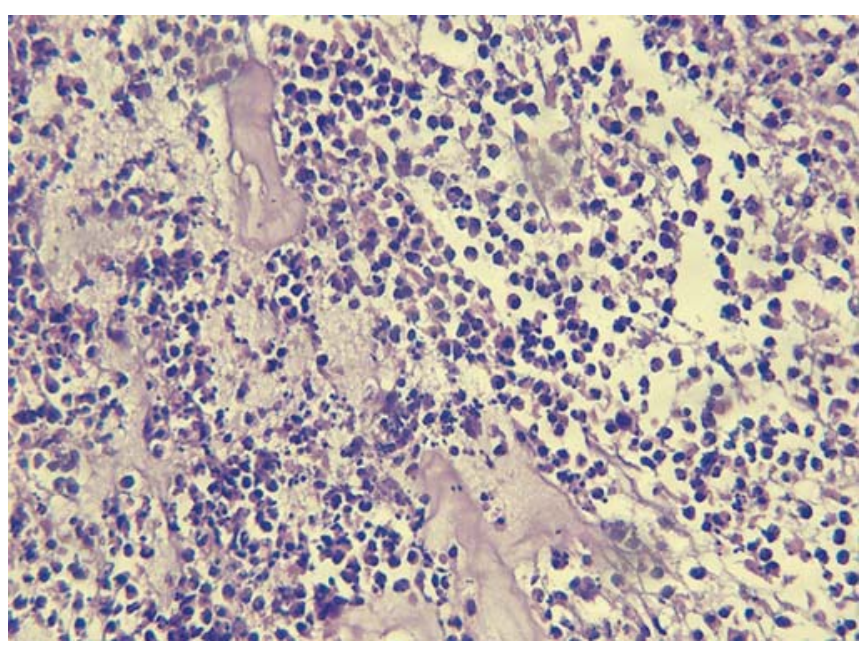

Fig. 2: HP showing sheet like proliferation of plasma cells of varying maturity and atypia and an eccentrically placed nucleus

The mass was excised under general anesthesia by lateral rhinotomy approach. Ant wall of maxilla was removed. M ass was completely removed from maxillary antrum. M ass had eroded the bony orbital floor. However, periosteum was found to be intact. Posterior wall of maxillary antrum was found to be partially deficient. M iddle turbinate and superior turbinated were excised. The excised mass was sent for HP study. HP shows sheet like proliferation of plasma cells of varying maturity and atypia and an eccentrically placed nucl eus. Immunohistochemical staining demonstrated the monoclonal nature of plasma cells. Patient is now under follow-up. At 6 months no recurrence has been noted.

\section{DISCUSSION}

The plasma cell dyscrasias are characterized by the clonal proliferation of plasma cells that produces a homogeneous immunoglobulin protein. The major plasma cell dyscrasias are as follows:

1. Multiple myeloma

2. Monoclonal gammopathy of unknown significance (MGUS)

3. Solitary plasmacytoma of bone

4. Extramedullary plasmacytoma and

5. Heavy chain disorders. ${ }^{8}$

Extramedullary plasmacytoma is a rare soft tissue malignant neoplasm that is made up of monoclonal plasma cells. It can be either primary (without evidence of disease in other foci) or part of a systemic process during the course of multiple myeloma.

The first case of extramedullary plasmacytoma was reported in 1905 by Scride. ${ }^{9}$ B etween then and 1997, more than 400 articles addressing extramedullary plasmacytoma were published. In 1999, A lexiou et al reviewed all previous reports of extramedullary plasmacytoma and found 869 cases; $714(82.2 \%)^{4}$ of them had occurred in the upper aerodigestive tract. The most frequently affected areas in the upper aerodigestive tract are the nasal cavity and PNS $(43.8 \%)$ followed by the nasopharynx $(18.3 \%)$, the oropharynx (17.8\%) and the larynx (11.1\%). ${ }^{4}$

Extramedullary plasmacytoma is more common in males (ratio 4:1) and $95 \%$ of cases occur in patients older than 40 years. ${ }^{10}$ Extramedullary plasmacytoma of the upper aerodigestive tract are most often occur as single lesions. ${ }^{1,8}$

The clinical presentation may vary according to the site of involvement. In sinonasal or nasopharyngeal involvement, the most common symptoms are mass, nasal obstruction and epistaxis. ${ }^{11}$ Other reported presenting symptoms include nasal discharge, pain, proptosis, cervical lymphadenopathy and cranial nerve palsy. ${ }^{10}$

The diagnosis of extramedullary plasmacytomas is usually after HP study. In addition, immunohistochemical study is used to differentiate extramedullary plasmacytomas from benign reactive plasmacytomas as well as other malignant disorders, such as undifferentiated carcinoma, melanoma and esthesioneuroblastoma. ${ }^{12}$

When a plasmacytoma is confirmed histologically, secondary diagnostic procedures must be carried to exclude systematic involvement. ${ }^{8}$

Malignant tumors of the nasal cavity (including extramedullary plasmacytomas) are treated by surgery and/ or radiotherapy. A lthough extramedullary plasmacytoma is known to be radio sensitive, most authors recommended a combination of surgery and radiotherapy for nasal cavity lesions. $^{13}$

The lesion in our patient was localized in the maxillary antrum and there was no sign of invasion into the underlying bone periosteum. We did not consider postoperative radiotherapy.

L ocal recurrence has been reported to occur in 8 to $30 \%$ of adequately treated cases within 3 to 61 months. ${ }^{2,3}$ Conversion of extramedullary plasmacytoma to multiple myeloma has been reported on 8 to $36 \%$ of cases. Therefore, long-term follow-up including both local and systemic surveillance is necessary. CT and measurement of serum immunoglobulin and Bence-J ones protein levels may be useful in detecting recurrence or conversion to multiple myeloma.

Although, several reports regarding extramedullary plasmacytoma in the nasal cavity have been published, a finding of such a lesion in the maxillary antrum is quite rare. 


\section{REFERENCES}

1. Balsaksi J G, M edeires J L, L umma M A, EL-N aggar A K. Plasma cell dyscrasias and the head and the neck. Ann Diagn Pathol 2002;6:129-40.

2. Knowling MA, Harwood AR, B ergsagol DE. Comparison of extramedullary plasmacytomas with solitary and multiple plasma cell tumors of bone. J Clin Oncol 1983;1:255-62.

3. Holland J, Trenker DA, W asserman TH, Fineberg B. plasmacytoma treatment results and conversion to myeloma. Cancer 1992;69:1513-17.

4. Alexiou C, Kau RJ, Dietzfelbinger $\mathrm{H}$, et al. Extramedullary plasmacytoma: Tumor occurrence and therapeutic concepts. Cancer 1999;85:2305-14.

5. Poole AG, M archetta FC. Extramedullary plasma cytoma of the head and neck. Cancer 1968;22:14-21.

6. Webb HE, Harrison EG, M asson JK, Remine WH. Solitary extramedullary myeloma (plasmacytoma) of the upper part of the respiratory tract and oropharynx. Cancer 1962;15:1142-55.

7. Fu Y S, Perzin KH. Nonepithelial tumors of the nasal cavity, paranasal sinuses and nasopharynx. A clinicopathological study. IX plasmacytomas. Cancer 1978;42:2399-406.

8. Lebotwitz RA, M orris L. Plasma cell dyscrasias and amyloidosis. Otolaryngology Clin North A m 2003;36:747-64.

9. Schridde H. W eitere U ntersuchungen uber dieK ornel unjen cler plasmazellen. Central bl Aiig Pathol A nat 1905;16:433-35.

10. K apadia SB, D esai U, C heng V S. Extramedullary plasmacytoma of the head and neck. A clinicopathological study of 20 cases. Medicine (Baltimore) 1982;61:317-29.
11. M iller FR, Lavertu P, W anamaker JR, et al. Plasmacytomas of the head and neck. Otolaryngol Head Neck Surg 1998;119: 614-18.

12. Komisar A, Schetman F, Da Silva M , et al. The histopathological diagnosis of head and neck tumors by spiral stains. Ear Nose Throat J 1989;68:705-6, 709-12.

13. Castro EB, Lewis JS, Strong EW. Plasmacytoma of paranasal sinuses and nasal cavity. A rch Otolaryngol 1973;97:326-29.

\section{ABOUT THE AUTHORS}

\section{Chinmaya Sundar Ray (Corresponding Author)}

A ssistant Professor, Department of ENT and Head-N eck Surgery, SCB

M edical College and Hospital, Cuttack, Odisha, India

\section{Aparajita Mishra}

A ssistant Professor, D epartment of Pathology, SCB M edical College and Hospital, Cuttack, Odisha, India

\section{Rabindra Kumar Khatua}

Senior Resident, Department of ENT, SCB M edical College and Hospital, Cuttack, Odisha, India

\section{Prasenjit Baliarsingh}

Postgraduate Student, Department of Otolaryngology, SCB M edical College and Hospital, Cuttack, Odisha, India 